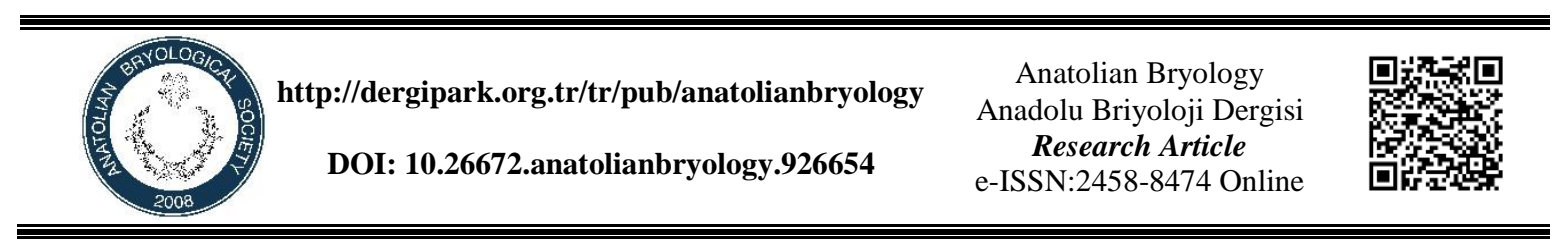

\title{
The Bryophyte Flora of Kümbet High Plateau (Dereli district, Turkey)
}

\author{
Hüseyin ERATA ${ }^{1}$ iD, Nevzat BATAN ${ }^{2 *}$ iD, Mevlüt ALATAŞ ${ }^{3}$ iD \\ ${ }^{1}$ Çanakkale Onsekiz Mart University, Bayramiç Vocational School, Çanakkale, TURKEY \\ ${ }^{2}$ Maçka Vocational School, Karadeniz Technical University, Trabzon, TURKEY \\ ${ }^{3}$ Tunceli Vocational School, Munzur University, Tunceli, TURKEY
}

\begin{abstract}
Received: 23 April 2021
Revised: 20 May 2021

Accepted: 27 May 2021

Abstract

As a result of bryological researches in the Kümbet High Plateau of Giresun province (Turkey), a total of 289 bryophyte taxa belonging to 131 genera and 64 families, of which are 50 liverworts and 239 mosses, were determined from 40 different localities. Among them, Neckera pumila and Pseudephemerum nitidum new to A4 grid square and all bryophyte taxa are new for Kümbet high plateau. Collected localities and substrata are presented at bryofloristic list.
\end{abstract}

Key words: Biodiversity, Bryophyte, Flora, Kümbet, Turkey.

\section{Kümbet Yaylası Briyofit Florası (Dereli, Türkiye)}

\section{$\ddot{O} \mathbf{z}$}

Giresun ili Kümbet Yaylası'nda yapılan briyolojik araştırmalar sonucunda, 40 farklı lokaliteden 64 familyaya ve 131 cinse ait, 50 ciğerotu ve 239 yapraklı karayosunu olmak üzere toplam 289 briyofit taksonu tespit edilmiştir. Neckera pumila ve Pseudephemerum nitidum A4 karesi için yenidir ve tüm briyofit taksonları Kümbet yaylası için yenidir. Toplanma lokaliteleri ve substratlar briyoflorostik listede verilmiştir.

Anahtar kelimeler: Biyoçeşitlilik, Briyofit, Flora, Kümbet, Türkiye

\section{Introduction}

Kümbet Plateau, which is located within the borders of Dereli district of Giresun province and it is surrounded by Giresun to the north, Yağlıdere and Keşap to the east, Şebinkarahisar to the south, and Bulancak to the west (Fig. 1).

The Giresun Mountains (e.g Kümbet High Plateau) belong to the East Black Sea Mountains and they are situated in the north east of Turkey. Kümbet High Plateau is located in the northern part of the Giresun
Mountains. The Giresun Mountains are very high and near the Black Sea. From the summit of these mountains, a lot of streams and rivers flow through deep valleys into the Black Sea. Oceanic climate prevails in the northern part of the Giresun Mountains. The mild, damp oceanic climate with high and evenly distributed rainfall makes bryodiversity very rich. Kümbet plateau, summers are warm and humid, and winters are cool, snowy, and damp (Anşin, 1981; Sesli et al., 2015; Erata et al., 2021).

\footnotetext{
* Corresponding author: nevzatbatan@gmail.com

(C) 2021 All rights reserved / Tüm haklarl saklıdır.

To cite this article: Erata H. Batan N. Alataş M. 2021. The Bryophyte Flora of Kümbet High Plateau (Dereli district, Turkey). Anatolian Bryology. 7:2,77-89.

(c) () \& 8 This work is licensed under a Creative Commons Attribution-Non Commercial 4.0 International License.
} 


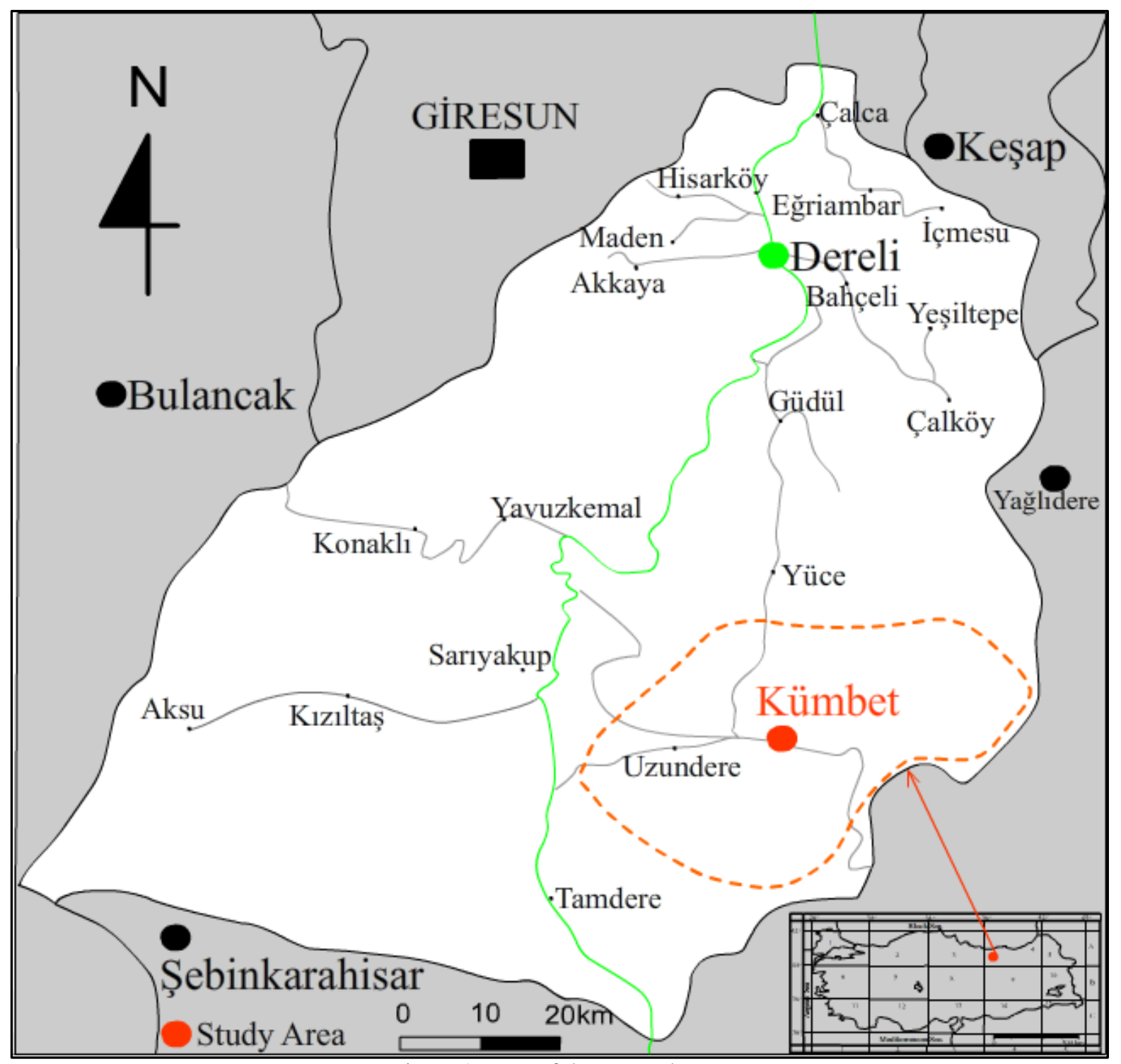

Figure 1. Map of the research area

Research area is covered mixed forest and the dominant taxa in the main vegetation are Alnus glutinosa (L.) Gaertn., Carpinus betulus L., C. orientalis Mill., Corylus avellana Thunb., Acer platanoides L., Tilia rubra DC, Ilex colchica Pojk., Daphne pontica L., Castanea sativa Mill., Fagus orientalis Lipsky, Picea orientalis (L.) Peterm., Pinus sylvestris L., Abies nordmanniana (Stev.) Spach. subsp.nordmanniana, Rhododendron luteum Sweet, $R$. ponticum L. Salix sp., Betula sp. and various Quercus (Anşin, 1981; Sesli et al., 2015; Erata et al., 2021).

\section{Materials and Methods}

The bryophyte samples were collected by authors in 40 different localities (Table 1) between 08 July 2019 and 30 August 2020 from Kümbet high plateau in Turkey (Fig. 1). The bryophyte samples were examined with stereomicroscope and light microscope. Identifications were determined by consulting various floras and keys (Crum and Anderson, 1981; Nyholm, 1986, 1989, 1993, 1998; Lewinsky, 1993; Blom, 1996; Smith, 2004; Paton, 1999; Pedrotti, 2001, 2006; Greven, 2003; Heyn and Herrnstadt, 2004; Frey et al., 2006; Guerra et al., 2006; 2010; 2014; 2018; Brugués et al., 2007; Kürschner and Frey, 2011). 


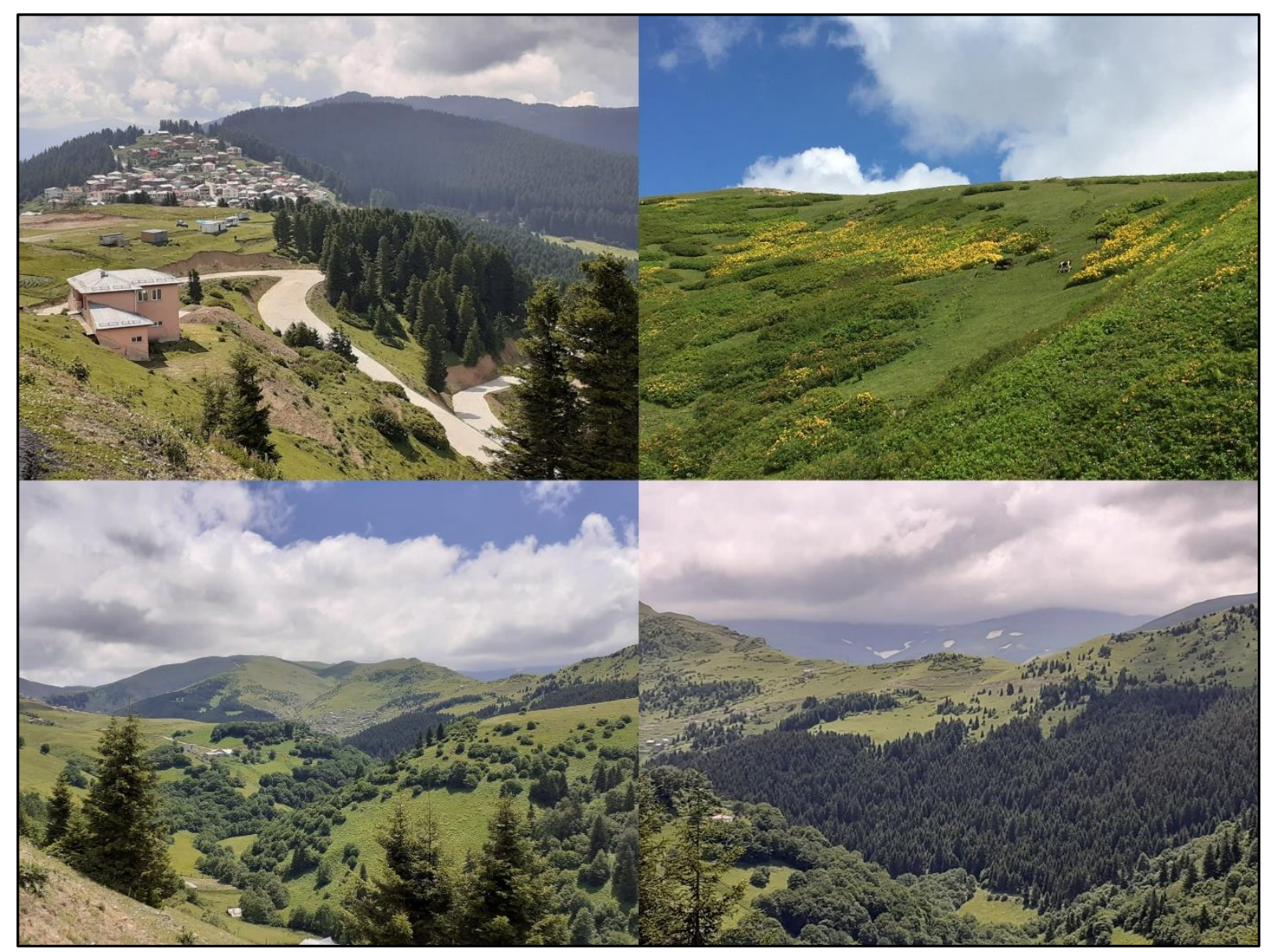

Figure 2. Some ecosystem types from Kümbet high plateau

Table 1. The collection localities.

\begin{tabular}{|c|c|c|c|}
\hline No & Locality & $\begin{array}{c}\text { Altitude Sea } \\
\text { Level }(m)\end{array}$ & Date \\
\hline 1 & $\begin{array}{l}\text { Kümbet: C1mbirtlık Forest Management warehouse-1, } \\
40^{\circ} 35^{\prime} 28^{\prime \prime} \mathrm{N} / 38^{\circ} 26^{\prime} 59^{\prime \prime} \mathrm{E}\end{array}$ & $1230-1260$ & 08.07 .2019 \\
\hline 2 & $\begin{array}{l}\text { Kümbet: Upper part of Cimbirtlik Forest Management } \\
\text { warehouse, } 40^{\circ} 35^{\prime} 08^{\prime \prime} \mathrm{N} / 38^{\circ} 26^{\prime} 43^{\prime \prime} \mathrm{E}\end{array}$ & $1270-1350$ & 08.07 .2019 \\
\hline 3 & $\begin{array}{l}\text { Kümbet: Upper part of Yüceköy, Southeastern hillside, } \\
40^{\circ} 34^{\prime} 56^{\prime \prime N} / 38^{\circ} 26^{\prime} 54^{\prime \prime} \mathrm{E}\end{array}$ & $1450-1420$ & 08.07 .2019 \\
\hline 4 & Kümbet: Armutalan high plateau, $40^{\circ} 34^{\prime} 17^{\prime \prime N} / 38^{\circ} 25^{\prime} 18^{\prime \prime E}$ & 1430 & 09.07.2019 \\
\hline 5 & $\begin{array}{l}\text { Kümbet: Samayil high plateau, } \\
40^{\circ} 35^{\prime} 41^{\prime \prime N} / 38^{\circ} 24^{\prime} 59^{\prime \prime E}\end{array}$ & $1800-1850$ & 09.07.2019 \\
\hline 6 & $\begin{array}{l}\text { Kümbet: Between Kümbet center and Keşoğ high plateau, } \\
40^{\circ} 35^{\prime} 41^{\prime \prime N} / 38^{\circ} 24^{\prime} 59^{\prime \prime E}\end{array}$ & $1780-1800$ & 09.07.2019 \\
\hline 7 & Kümbet: Aymaç Hill, $40^{\circ} 34^{\prime} 04^{\prime \prime} \mathrm{N} / 38^{\circ} 25^{\prime} 41^{\prime \prime E}$ & $1810-1850$ & 09.07 .2019 \\
\hline 8 & $\begin{array}{l}\text { Kümbet: Between Kümbet Kümbet center and Ş1h high } \\
\text { plateau, 2. Km, } 40^{\circ} 33^{\prime} 20^{\prime \prime} \mathrm{N} / 38^{\circ} 28^{\prime} 28^{\prime \prime} \mathrm{E}\end{array}$ & $1720-1730$ & 09.07.2019 \\
\hline 9 & Kümbet: Başoba high plateau, $40^{\circ} 31^{\prime} 48^{\prime \prime N} / 38^{\circ} 29^{\prime} 01^{\prime \prime E}$ & $2000-2050$ & 10.07.2019 \\
\hline 10 & Kümbet: Çeğeril high plateau, $40^{\circ} 33^{\prime} 35^{\prime \prime N} / 38^{\circ} 29^{\prime} 20^{\prime \prime} \mathrm{E}$ & 1927 & 10.07 .2019 \\
\hline 11 & $\begin{array}{lclll}\text { Kümbet: Koç Kayas1 } & \text { Nature } & \text { Park } & -1, \\
40^{\circ} 34^{\prime} 58^{\prime \prime} \mathrm{N} / 38^{\circ} 29^{\prime} 02^{\prime \prime} \mathrm{E} & & & & \\
\end{array}$ & $2110-2130$ & 10.07.2019 \\
\hline 12 & $\begin{array}{lcccc}\text { Kümbet: } & \text { Koç } & \text { Kayas1 } & \text { Nature } & \text { Park-2, } \\
40^{\circ} 33^{\prime} 50^{\prime \prime N} / 38^{\circ} 28^{\prime} 47^{\prime \prime E} & & & \\
\end{array}$ & 1910-1950 & 10.07.2019 \\
\hline 13 & Kümbet: Köyyeri high plateau, $40^{\circ} 31^{\prime} 28^{\prime \prime N} / 38^{\circ} 26^{\prime} 29^{\prime \prime E}$ & 1620 & 11.07 .2019 \\
\hline 14 & Kümbet: Homorlu high plateau, $40^{\circ} 32^{\prime} 49^{\prime \prime} \mathrm{N} / 38^{\circ} 26^{\prime} 14^{\prime \prime} \mathrm{E}$ & 1600 & 11.07 .2019 \\
\hline 15 & Kümbet: Elemeği high plateau, $40^{\circ} 31^{\prime} 59^{\prime \prime N} \mathrm{~N} / 38^{\circ} 25^{\prime} 39^{\prime \prime} \mathrm{E}$ & $1910-1930$ & 11.07.2019 \\
\hline
\end{tabular}




\begin{tabular}{|c|c|c|c|}
\hline 16 & Kümbet: Uzundere village $-1,40^{\circ} 32^{\prime} 41^{\prime \prime} \mathrm{N} / 38^{\circ} 24^{\prime} 32^{\prime \prime} \mathrm{E}$ & $1610-1650$ & 11.07 .2019 \\
\hline 17 & Kümbet: Uzundere village $-2,40^{\circ} 32^{\prime} 54^{\prime \prime N} / 38^{\circ} 21^{\prime} 52^{\prime \prime} \mathrm{E}$ & 1330 & 11.07.2019 \\
\hline 18 & $\begin{array}{lccc}\text { Kümbet: } & \text { Road } \\
40^{\circ} 34^{\prime} 59^{\prime \prime} \mathrm{N} / 38^{\circ} 24^{\prime} 50^{\prime \prime} \mathrm{E} & \text { the } & \text { Yeşilvadi-Kümbet } & -1, \\
\end{array}$ & 1570 & 12.07.2019 \\
\hline 19 & Kümbet: Kümbet village, $40^{\circ} 33^{\prime} 04^{\prime \prime N} / 38^{\circ} 27^{\prime} 18^{\prime \prime E}$ & $1610-1630$ & 12.07 .2019 \\
\hline 20 & Kümbet: Yağmurca high plateau, $40^{\circ} 32^{\prime} 15^{\prime \prime} \mathrm{N} / 38^{\circ} 28^{\prime} 21^{\prime \prime E}$ & 1875 & 12.07 .2019 \\
\hline 21 & $\begin{array}{l}\text { Kümbet: Road the Yeşilvadi-Kümbet }-2, \\
40^{\circ} 34^{\prime} 36^{\prime \prime N} / 38^{\circ} 24^{\prime} 60^{\prime \prime} \mathrm{E}\end{array}$ & 1720 & 12.09.2019 \\
\hline 22 & 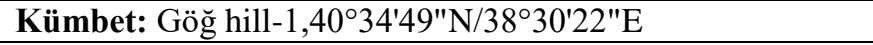 & 2320 & 12.09 .2019 \\
\hline 23 & $\begin{array}{l}\text { Kümbet: Koç Kayası Nature Park, inside the social facility, } \\
40^{\circ} 34^{\prime} 12^{\prime \prime} \mathrm{N} / 38^{\circ} 28^{\prime} 49^{\prime \prime} \mathrm{E}\end{array}$ & 1850 & 13.09.2019 \\
\hline 24 & Kümbet: Göğ hill-2, 40³4'50"N/38³0'22"E & 2370 & 13.09 .2019 \\
\hline 25 & $\begin{array}{l}\text { Kümbet: Enter the Kuzu lake high plateau, } \\
40^{\circ} 35^{\prime} 01^{\prime \prime N} / 38^{\circ} 29^{\prime} 14^{\prime \prime} \mathrm{E}\end{array}$ & 2080 & 13.09.2014 \\
\hline 26 & Kümbet: Ş, Sh high plateau $-2,40^{\circ} 31^{\prime} 45^{\prime \prime} \mathrm{N} / 38^{\circ} 29^{\prime} 07^{\prime \prime} \mathrm{E}$ & 1980 & 14.09 .2019 \\
\hline 27 & Kümbet: Şı high plateau $-3,40^{\circ} 31^{\prime} 17^{\prime \prime N} / 38^{\circ} 29^{\prime} 29^{\prime \prime} \mathrm{E}$ & 2050 & 14.09 .2019 \\
\hline 28 & $\begin{array}{l}\text { Kümbet: Cimbirtllk Forest Management warehouse-2, } \\
40^{\circ} 35^{\prime} 19^{\prime \prime N} / 38^{\circ} 27^{\prime} 14^{\prime \prime E}\end{array}$ & 1350 & 26.06.2020 \\
\hline 29 & $\begin{array}{l}\text { Kümbet: Eskioba high plateau, } \\
40^{\circ} 36^{\prime} 05^{\prime \prime} \mathrm{N} / 38^{\circ} 25^{\prime} 07^{\prime \prime} \mathrm{E}\end{array}$ & 1792 & 26.06 .2020 \\
\hline 30 & $\begin{array}{l}\text { Kümbet: Şh high plateau exit, } \\
40^{\circ} 32^{\prime} 44^{\prime \prime} / 38^{\circ} 30^{\prime} 41^{\prime \prime E}\end{array}$ & $1810-1850$ & 27.06.2020 \\
\hline 31 & $\begin{array}{l}\text { Kümbet: Enter thr Göbel high plateau, } \\
40^{\circ} 33^{\prime} 15^{\prime \prime} \mathrm{N} / 38^{\circ} 29^{\prime} 04^{\prime \prime} \mathrm{E}\end{array}$ & $1830-1900$ & 27.06.2020 \\
\hline 32 & $\begin{array}{l}\text { Kümbet: Erik stream high plateau, } \\
40^{\circ} 30^{\prime} 53^{\prime \prime} \mathrm{N} / 38^{\circ} 26^{\prime} 48^{\prime \prime} \mathrm{E}\end{array}$ & 1753 & 28.06.2020 \\
\hline 33 & Kümbet: Sirganlı high plateau, $40^{\circ} 31^{\prime} 28^{\prime \prime N} / 38^{\circ} 25^{\prime} 17^{\prime \prime E}$ & 1981 & 29.06 .2020 \\
\hline 34 & $\begin{array}{l}\text { Kümbet: Upper part of Uzundere village, } \\
40^{\circ} 33^{\prime} 52^{\prime \prime} \mathrm{N} / 38^{\circ} 25^{\prime} 24^{\prime \prime} \mathrm{E}\end{array}$ & 1820 & 30.06 .2020 \\
\hline 35 & $\begin{array}{l}\text { Kümbet: Between Goruk high plateau and Giran high } \\
\text { plateau, } 40^{\circ} 34^{\prime} 38^{\prime \prime} \mathrm{N} / 38^{\circ} 24^{\prime} 52^{\prime \prime} \mathrm{E}\end{array}$ & 1710 & 24.08 .2020 \\
\hline 36 & $\begin{array}{l}\text { Kümbet: Tekke high plateau, } \\
40^{\circ} 32^{\prime} 22^{\prime \prime} \mathrm{N} / 38^{\circ} 28^{\prime} 09^{\prime \prime} \mathrm{E}\end{array}$ & 1820 & 25.08 .2020 \\
\hline 37 & $\begin{array}{l}\text { Kümbet: Lower part of Şih high plateau, } \\
40^{\circ} 32^{\prime} 52^{\prime \prime} \mathrm{N} / 38^{\circ} 29^{\prime} 29^{\prime \prime} \mathrm{E}\end{array}$ & 1750 & 26.08.2020 \\
\hline 38 & $\begin{array}{l}\text { Kümbet: Başçatak high plateau, } \\
40^{\circ} 30^{\prime} 21^{\prime \prime N} / 38^{\circ} 26^{\prime} 56^{\prime \prime E}\end{array}$ & $1850-1880$ & 27.08.2020 \\
\hline 39 & $\begin{array}{l}\text { Kümbet: Between Tekke high plateau and Yağmurca high } \\
\text { plateau, } 40^{\circ} 33^{\prime} 02^{\prime \prime N} / 38^{\circ} 27^{\prime} 08^{\prime \prime E}\end{array}$ & 1616 & 29.08 .2020 \\
\hline 40 & $\begin{array}{l}\text { Kümbet: Road the Yeşilvadi- Kümbet -3, } \\
40^{\circ} 35^{\prime} 12^{\prime \prime} \mathrm{N} / 38^{\circ} 24^{\prime} 11^{\prime \prime} \mathrm{E}\end{array}$ & 1465 & 30.08 .2020 \\
\hline
\end{tabular}

Nomenclature of the species and synonym status were evaluated by reviewing Hodgetts et al. (2020). The situation of bryophyte taxa was appraised by reviewing related literature for Giresun Province (Özdemir and Batan, 2017a; Erata et al., 2018). The status of bryophyte taxa was evaluated by reviewing related literature for the A4 square (Abay et al., 2016; Özdemir and Batan, 2017 a, b; Erata et al., 2018). Bryophyte samples are deposited in the private herbarium of Hüseyin ERATA at the Bayramiç vocational school, Çanakkale Onsekiz Mart University (Çanakkale), Turkey.

\section{Results and Discussion}

\subsection{Results}

As a result of the study, 50 liverwort taxa (belonging to 21 families and 30 genera), 239 moss taxa (belonging to 43 families and 101 genera) and a total of 289 bryophyte taxa (belonging to 64 families and 131 genera) were determined.

For each taxa, their localities and substrate were given in the floristic list. Taxa for new Giresun province is indicated with (\#) bryofloristic list. Also new for A4 square is indicated with $(+)$ in the bryofloristic list.

\section{Bryofloristic list}

Marchantiophyta

Anastrophyllaceae L.Söderstr., De Roo \& Hedd.

Barbilophozia Loeske

Barbilophozia barbata (Schmidel ex Schreb.) Loeske Loc.: 11, 15, 25, 33; on rock, on soil.

B. hatcheri (A. Evans) Loeske - Loc.: 23; on rock. \#B. lycopodioides (Wallr.) Loeske - Loc.: 19; on rock. 
\#B. sudetica (Nees ex Huebener) L.Söderstr., De Roo $\&$ Hedd. (Syn: Lophozia sudetica (Nees ex Huebener) Grolle) - Loc.: 13; on a dead tree trunk.

Gymnocolea (Dumort.) Dumort.

Gymnocolea inflata (Huds.) Dumort - Loc.: 12; on wet soil.

Sphenolobus (Lindb.) Berggr.

Sphenolobus minutus (Schreb.) Berggr. (Syn: Anastrophyllum minutum (Schreb.) Schust.) - Loc.: 11, 12; on soil.

Cephalozieaceae Mig.

Cephalozia (Dumort.) Dumort.

Cephalozia bicuspidata (L.) Dumort. - Loc.: 15, 16, 22, 26, 33, 39; on soil, on wet soil.

Cephaloziellaceae Douin

Cephaloziella (Spruce) Schiffn.

Cephaloziella divaricata (Sm.) Schiffn. - Loc.: 22; on wet soil.

Lophoziaceae Cavers

Lophozia (Dumort.) Dumort.

\#Lophozia ventricosa (Dicks.) Dumort. - Loc.: 11, 12, 39; on soil, on a dead tree trunk.

Lophoziopsis Konstant. \& Vilnet

Lophoziopsis longidens (Lindb.) Konstant. \& Vilnet (Syn: Lophozia longidens (Lindb.) Macoun) - Loc.: 12, 26; on wet soil, on a dead tree trunk.

Tritomaria Loeske

\#Tritomaria exsecta Schmidel ex. Schrad.) Loeske. Loc.: 6, 21, 29, 40; on dead tree trunk.

Trilophozia (RMSchuster) VA Bakalin

\#Trilophozia quinquedentata (Huds.) Bakalin (Syn: Tritomaria quinquedentata (Huds.) H. Buch.) - Loc.: 12; on a dead tree trunk.

Scapaniaceae Mig.

Diplophyllum (Dumort.) Dumort.

Diplophyllum albicans (L.) Dumort. - Loc.: 12; on wet soil.

D. taxifolium (Wahlenb) Dumort - Loc.: 11, 12, 25, 29; on wet soil.

Scapania (Dumort.) Dumort.

\#Scapania aequiloba (Schwäagr.) Dumort. - Loc.: 11; on wet soil.

S. irrigua (Ness) Ness - Loc.: 13, 18, 22, 23, 32, 35; on wet soil.

\#S. subalpina (Nees ex Lindenb.) Dumort. - Loc.: 13; on wet soil.

S. undulata (L.) Dumort. - Loc.: 19, 36; on wet soil.

Schistochilopsis (N. Kitag.) Konst.

Schistochilopsis incisa (Schrad.) Konstant. (Syn:

Lophozia incisa (Schrad.) Dumort. - Loc.: 12, 35; on a dead tree trunk

Calypogeiaceae Arnell

Calypogeia Raddi

Calypogeia fissa (L.) Raddi. - Loc.: 22; on wet soil.

C. muelleriana (Schiffn.) Müll. Frib. - Loc.: 22, 26; on wet soil.

Jungermanniaceae Rchb.

Jungermannia L.

Jungermannia atrovirens Dumort. - Loc.: 6, 37; on wet soil.
Solenostomataceae Stotler \& Crand.-Stotl.

Solenostoma Mitt. emend. Zerov

Solenostoma gracillimum (Sm.) R.M.Schust. (Syn: Jungermannia gracillima Sm.) - Loc.: 9, 26; on wet soil.

\#S. hyalinum (Lyell) Mitt. (Syn: Jungermannia hyalina Lyell) - Loc.: 9, 11, 12; on wet soil.

S. sphaerocarpum (Hook.) Steph. Sp. Hepat (Syn: Jungermannia sphaerocarpa Kanca.) - Loc.: 11, 12, 13,22 ; on wet soil, on wet rock.

Blepharostomataceae W.Frey \& M.Stech

Blepharostoma (Dumort. Emend. Lindb.) Dumort.

\#Blepharostoma trichophyllum (L.) Dumort. - Loc.: 1, $3,4,5,6,8,12,29,35,40$; on calcareous rock, on a dead tree trunk.

Lepidoziaceae Limpr.

Lepidozia (Dumort.) Dumort.

\#Lepidozia reptans (L.) Dumort. - Loc.: 3, 6, 40; on a dead tree trunk.

Lophocoleaceae Vanden Berghen

Chiloscyphus Corda

Chiloscyphus pallescens (Ehrh. ex Hoffm.) Dumort. Loc.: 8; on wet soil.

C. polyanthos (L.) Corda - Loc.: 9, 12, 26, 28, 35; on wet soil.

Lophocolea (Dumort.) Dumort.

Lophocolea bidentata (L.) Dumort. - Loc.: 3, 6, 10, 14, $15,16,18,31,33,35$; on wet soil, on rock, on a dead tree trunk.

L. heterophylla (Schrad.) Dumort. - Loc.: 1, 3, 4, 5, 7, $8,10,12,16,18,22,29,31,34,35$; on soil, on a dead tree trunk.

L. minor Nees. - Loc.: 1, 7, 12, 16, 40; on soil, on a dead tree trunk.

Plagiochilaceae Müll. Frib.

Pedinophyllum (Lindb.) Lindb.

Pedinophyllum interruptum (Nees) Kaal. - Loc.: 3, 6, $11,12,13,15,16,19,20,22,24,25,26,28,31,33,35$, $36,38,40$; on wet soil, on wet rock.

Plagiochila (Dumort.) Dumort.

Plagiochila asplenioides (L. emend. Taylor) Dumort. Loc.: 1, 3, 6, 8, 10, 12, 13, 14, 15, 18, 19, 20, 22, 28, $29,31,32,33,35,36,39,40$; on soil, on rock, on a dead tree trunk.

P. porelloides (Torrey ex Nees) Lindenb. - Loc.: 1, 2, $7,8,9,11,12,18,19,20,21,25,35,36,40$; on soil, on rock.

Myliaceae Schljakov

Mylia Gray

Mylia taylorii (Hook.) Gray. - Loc.: 12, 19, 22; on wet soil.

Frullaniaceae Lorch

Frullania Raddi

Frullania dilatata (L.) Dumort. - Loc.: 1, 2, 7, 8, 36, 39; on rock, on tree bark.

F. tamarisci (L.) Dumort. - Loc.: 1, 3, 13, 17, 28, 29,

32, 38; on tree bark.

Porellaceae Cavers

Porella L. 
Porella cordaeana (Huebener) Moore - Loc.: 10, 31; on rock, on tree bark.

P. platyphylla (L.) Pfeiff. - Loc.: 2, 3, 37, 39; on rock, on tree bark.

Radulaceae Müll. Frib.

Radula Dumort.

Radula complanata (L.) Dumort. - Loc.: 1, 11, 19, 20, 29, 35, 36; on rock, on tree bark.

R. lindenbergiana Gottsche ex C. Hartm. - Loc.: 1, 6, $8,10,13,15,16,22,24,29,31,33,35$; on rock, on tree bark.

Aneuraceae H. Klinggr.

Riccardia Gray

\#Riccardia multifida (L.) Gray - Loc.: 14; on wet soil. \#R. palmata (Hedw.) Carruth. - Loc.: 8; on wet soil.

Metzgeriaceae H. Klinggr.

Metzgeria Raddi

Metzgeria conjugata Lindb. - Loc.: 8.; on soil.

M. furcata (L.) Dumort. - Loc.: 1; on rock.

Pelliaceae H. Klinggr

Pellia Raddi

Apopellia endiviifolia (Dicks.) Nebel \& D.Quandt. (Syn: Pellia endiviifolia (Dicks.) Dumort) - Loc.: 4, 5, 18, 28; on wet soil.

Pellia epiphylla (L.) Corda - Loc.: 1, 3, 5, 6, 10, 12, 19, 22, 23, 31, 36; on wet soil.

Conocephaleaceae Müll. Frib. Ex Grolle

Conocephalum Hill

Conocephalum conicum (L.) Dumort. - Loc.: 1, 2, 3, 6, $9,17,21,28,39,40$; on wet rock and on wet

Marchantiaceae Lindl.

Marchantia L.

Marchantia polymorpha L. - Loc.: 6, 7, 10, 13, 16, 18, 19, 20, 29, 30, 31, 32, 35, 36, 37, 38; on wet soil.

Bryophyta

Sphagnaceae Dumort.

Sphagnum L.

Sphagnum auriculatum Schimp. - Loc.: 25, 26; on wet soil in bog.

\#S. fallax (H. Klinggr.) H. Klinggr. - Loc.: 26, 27; on wet soil in bog.

$S$. inundatum Russow - Loc.: 26; on wet soil in bog. \#S. platyphyllum (Lindb. ex Braithw.) Warnst. - Loc.: 23, 25, 26; on wet soil in bog.

\#S. squarrosum Crome - Loc.: 26; on wet soil in bog.

S. subsecundum Nees - Loc.: 26, 27; on wet soil in bog. \#S. teres (Schimp.) Ångstr. - Loc.: 26; on wet soil in bog.

Polytrichaceae Schwagr.

Atrichum P.Beauv.

\#Atrichum tenellum (Röhling) Bruch \& Schimper. -

Loc.: 4, 9; on soil.

A. undulatum (Hedw.) P. Beauv. - Loc.: 1, 2, 4, 5, 6, 7, 9, 13, 18, 21, 28, 29, 31, 35, 39, 40; on soil.

Pogonatum P.Beauv.

Pogonatum aloides (Hedw.) P. Beauv. - Loc.: 1, 7, 8, 10, 13, 16, 19, 20, 30, 31, 32, 35; on soil, on rock.

P. urnigerum (Hedw.) P.Beauv. - Loc.: 3, 4, 5, 9, 14, 17, 28; on soil, on rock.

Polytrichastrum Hedw.
\#Polytrichastrum alpinum (Hedw.) G.L.Sm. - Loc.: 2, 7; on soil.

Polytrichum Hedw.

Polytrichum commune Hedw. - Loc.: 1, 4, 8, 12, 13, 18, 26, 35; on soil.

P. formosum Hedw. - Loc.: 2, 3, 9, 12, 13, 14, 17, 19, 22, 26, 29, 32, 35, 36, 38, 39, 40; on soil, on rock.

$P$. juniperinum Hedw. - Loc.: 9, 10, 13, 15, 16, 23, 24,

29, 30, 31, 32, 33, 38; on soil, on rock.

P. piliferum Hedw. - Loc.: 9, 10, 11, 12, 13, 22, 24, 25,

31, 32; on soil, on rock.

Tetraphidaceae Schimp.

Tetraphis Hedw.

Tetraphis pellucida Hedw. - Loc.: 1, 3, 40; on a dead tree trunk.

Encalyptaceae Schimp.

Encalypta Dixon

\#Encalypta ciliata Hedw. - Loc.: 8, 14, 15, 33, 39; on rock.

E. streptocarpa Hedw. - Loc.: 16, 17; on rock.

E. vulgaris Hedw. - Loc.: 8, 14; on rock.

Funariaceae Schwagr.

Funaria Hedw.

Funaria hygrometrica Hedw. - Loc.: 11, 18, 35; on soil.

Hymenolomataceae Ignatov \& Fedosov

Hymenoloma Dusén

Hymenoloma compactum (Schwägr.) Ochyra - Loc.:

17 ; on rock.

H. crispulum (Hedw.) Ochyra. - Loc.: 11, 15, 22, 25, 33; on soil, on rock.

Flexitrichaceae Ignatov \& Fedosov

Flexitrichum Ignatov \& Fedosov

\#Flexitrichum flexicaule (Schwägr.) Ignatov \& Fedosov (Syn: Ditrichum flexicaule (Schwägr.) Hampe) - Loc.: 11, 29, 30; on soil, on rock.

\#F. gracile (Mitt.) Ignatov \& Fedosov (Syn: Ditrichum gracile (Mitt.) Kuntze.) - Loc.: 9, 14; on soil, on rock.

Leucobryaceae Schimp.

Dicranodontium Bruch \& Schimp.

Dicranodontium denudatum (Brid.) E. Britton. - Loc.: $1,3,28$; on a dead tree trunk.

Aongstroemiaceae De Not.

Dichodontium Schimp.

Dichodontium pellucidum (Hedw.) Schimp. - Loc.:1, 3, 4, 7, 11, 12, 25, 28, 29, 39; on soil

Diobelonella Ochyra.

Diobelonella palustris (Dicks.) Ochyra (Syn: Dichodontium palustre (Dicks.) M. Stech.) - Loc.: 5, 9, 22, 26; on soil.

Dicranellaceae M.Stech

Dicranella (Müll.Hal.) Schimp.

Dicranella heteromalla (Hedw.) Schimp. - Loc.: 1, 5, 7, 13, 32; on soil.

\#D. rufescens (Dicks.) Schimp. - Loc.: 26; on soil.

Fissidentaceae Schimp.

Fissidens Hedw.

Fissidens adianthoides Hedw. - Loc.: 8, 12, 22; on soil, on the rock cracks. 
F. dubius P. Beauv. - Loc.: 2, 9, 11, 25, 29; on wet soil, on the rock cracks.

\#F. taxifolius Hedw. - Loc.: 1, 3, 5, 6, 9, 29, 31, 37; on soil, on the rock cracks.

Dicranaceae Schimp.

Dicranum Hedw.

Dicranum bonjeanii De Not. - Loc.: 1, 22; on soil.

D. majus Turner. - Loc.: 29; on soil.

D. polysetum Sw. ex anon. - Loc.: 34; on soil.

D. scoparium Hedw. - Loc.: 1, 2, 3, 4, 5, 6, 7, 8, 9, 11 ,

$12,13,15,18,19,23,25,29$,

$32,33,34,35,36,38,39$; on soil, on rock, on a dead tree trunk.

\#D. spadiceum J.E.Zetterst. - Loc.:11, 15, 24, 25, 26; on soil.

Rhabdoweisiaceae Limpr.

Cynodontium Bruch \& Schimp.

Cynodontium jenneri (Schimp.) Stirt. - Loc.: 29; on rock.

Dicranoweisia Milde.

Dicranoweisia cirrata (Hedw.) Lindb. - Loc.: 8, 12; on soil.

Kiaeria I. Hagen

Kiaeria starkei (F. Weber \& D. Mohr) I. Hagen - Loc.: 12; on soil.

Ditrichaceae Limpr.

Ceratodon Brid.

Ceratodon purpureus (Hedw.) Brid. - Loc.: 1, 2, 5, 6, $12,13,15,16,17,21,23,29,32,38,40$; on soil; on rock.

Pseudephemerum (Lindb.) I.Hagen

+Pseudephemerum nitidum (Hedw.) Loeske - Loc.: 9; on wet soil.

Pottiaceae Schimp.

Anoectangium Schwägr.

Anoectangium aestivum (Hedw.) Mitt. - Loc.: 12, 16, 17,38 ; on rock.

Bryoerythrophyllum P.C.Chen.

Bryoerythrophyllum recurvirostrum (Hedw.) P.C. Chen. - Loc.: 11, 14, 17, 25, 38; on rock.

\section{Chionoloma Dixon}

Chionoloma tenuirostre (Hook. \& Taylor) M.Alonso, MJCano \& JAJiménez (Syn: Oxystegus tenuirostris (Hook. \& Taylor) A.J.E.Sm.) - Loc.: 1, 4; on rock.

Didymodon Hedw.

Didymodon ferrugineus (Schimp. ex Besch.) M.O.Hill.

- Loc.: 1, 2; on soil, on rock.

D. luridus Hornsch. - Loc.: 38; on rock.

D. tophaceus (Brid.) Lisa. - Loc.: 1; on rock.

D. vinealis (Brid.) R.H.Zander- Loc.: 1; on rock.

Syntrichia Brid.

Syntrichia montana Nees - Loc.: 14, 30, 39; on soil, on rock.

S. norvegica F.Weber - Loc.: 38; on rock.

S. ruraliformis (Besch.) Mans. (Syn: S. ruralis (Hedw.) F.Weber \& D.Mohr var. ruraliformis

(Besch.) Delogne) - Loc.: 16, 19, 20, 38; on soil, on rock.
S. ruralis (Hedw.) F.Weber \& D.Mohr - Loc.: 2, 10, $11,13,15,19,23,25,30,31,32,33,36$; on soil, on rock

Syntrichia virescens (De Not.) Ochyra - Loc.: 17; on rock.

Tortella (Müll.Hal.) Limpr.

Tortella fragilis (Hook. \& Wilson) Limpr. - Loc.: 7; on rock.

\#T. humilis (Hedw.) Jenn. - Loc.: 28; on rock.

\#T. nitida (Lindb.) Broth. - Loc.: 3, 6, 7, 11; on soil, on rock.

T. tortuosa (Hedw.) Limpr. - Loc.: 1, 2, 4, 8, 10, 11, $12,15,16,17,22,25,28,30,31,33,37,38,39$; on soil, on rock.

Tortula Hedw.

Tortula marginata (Bruch \& Schimp.) Spruce. - Loc.: 2, 6, 10, 18, 31, 35, 39, 40; on soil, on rock.

\#T. mucronifolia Schwägr - Loc.: 15, 17; on rock.

T. subulata Hedw. - Loc.: 1, 4, 5, 7, 8, 11, 15, 25, 29, $30,33,38$; on soil, on rock.

Weissia Hedw.

\#Weissia brachycarpa (Nees \& Hornsch.) Jur. - Loc.: 7; on soil.

W. controversa Hedw. - Loc.: 8, 11, 22, 25; on soil.

Saelaniaceae Ignatov \& Fedosov

Saelania Lindb.

\#Saelania glaucescens (Hedw.) Broth. - Loc.: 7, 11, 25; on rock.

Grimmiaceae Arn.

Grimmia Hedw.

\#Grimmia anomala Hampe ex Schimp. - Loc.: 14; on rock.

G. alpestris (Weber \& Mohr) Schleicher. - Loc.: 13, 15, 17, 22, 24, 30, 32, 33, 38; on rock.

G. dissimulata E.Maier. - Loc.: 10, 24, 31; on rock.

\#G. elatior Bruch ex Bals.-Criv. \& De Not. - Loc.: 11, 25,30 ; on rock.

G. elongata Kaulf. - Loc.: 24; on rock.

\#G. funalis (Schwaegr.) Bruch \& Schimp. - Loc.: 4, 11, 17, 25, 30, 38; on rock.

G. hartmannii Schimp. - Loc.: 2, 8, 10, 11, 12, 15, 16, 22, 23, 24, 25, 30, 31, 33; on rock.

G. longirostris Hook. - Loc.: 11, 15, 25; on rock.

G. montana Bruch \& Schimp. - Loc.: 9, 38; on rock.

G. muehlenbeckii Schimp. - Loc.: 38; on rock.

G. ovalis (Hedw.) Lindb. - Loc.: 13, 32; on rock.

G. pulvinata (Hedw.) Sm. - Loc.: 14; on rock.

Racomitrium Brid.

Racomitrium aciculare (Hedw.) Brid. - Loc.: 8; on rock.

R. affine (F. Weber \& D. Mohr) Lindb. - Loc.: 13, 32; on rock.

R. aquaticum (Brid. ex Schrad.) Brid. - Loc.: 12; on rock.

R. canescens (Hedw.) Brid. - Loc.: 11, 13, 15, 19, 20, 25, 32, 33, 36; on rock.

R. elongatum Ehrh. ex Frisvoll - Loc.: 9, 16, 19, 23, 29, 36, 40; on rock.

$R$. ericoides Brid. - Loc.: 2, 10, 16, 22, 24, 29, 31, 34; on rock. 
R. sudeticum (Funck) Bruch \& Schimp. - Loc.: 4, 19, 20, 36; on rock.

Schistidium Bruch \& Schimp.

Schistidium apocarpum (Hedw.) Bruch \& Schimp. -

Loc.: 2, 8, 19, 20, 28, 36; on rock.

S. confertum (Funck) Bruch \& Schimp. - Loc.: 2, 10, 11, 17, 25, 31, 30, 38; on rock.

S. crassipilum H.H.Blom - Loc.: 2, 19; on rock. \#S. dupretii (Thér.) W.A.Weber - Loc.: 10, 31, 39; on rock.

S. elegantulum H.H.Blom - Loc.: 13, 17, 32; on rock. S. flaccidum H.H.Blom - Loc.: 11, 39; on rock. \#S. helveticum (Schkuhr) Deguchi - Loc.: 9; on rock. S. papillosum Culm. - Loc.: 2, 4, 11, 14, 15, 16, 17, 25, 33; on rock.

S. platyphyllum (Mitt.) H.Perss. - Loc.: 14; on rock. S. rivulare (Brid.) Podp. - Loc.: 11, 15, 19, 33; on rock. S. trichodon (Brid.) Poelt - Loc.: 2, 9; on rock.

Hedwigiaceae Schimp.

Hedwigia P.Beauv.

Hedwigia ciliata (Hedw.) P.Beauv. var. ciliata - Loc.: $11,13,14,15,17,25,30,32,33$; on rock.

H. emodica Hampe ex Müll.Hal. (Syn: H. ciliata var. leucophaea Bruch \& Schimp. - Loc.: 11, 23, 25, 38; on rock.

Bartramiaceae Schwagr.

Bartramia Hedw.

Bartramia halleriana Hedw. - Loc.: 1, 8, 11, 14, 16, $19,20,25,36,39$; on wet rock, on the rock cracks.

\#B. ithyphylla Brid. - Loc.: 11, 12, 14, 15, 19, 20, 25, $30,33,36,38,39$; on rock, on the rock cracks.

B. pomiformis Hedw - Loc.: 12; on the rock cracks.

\section{Philonotis Brid.}

Philonotis caespitosa Jur. - Loc.: 9, 13, 32; near stream, on wet soil.

P. calcarea (Bruch \& Schimp.) Schimp. - Loc.: 9, 10, 13, 29, 31, 32; near stream, on wet soil, on wet rock.

P. fontana (Hedw.) Brid. - Loc.: 1, 5, 9, 13, 18, 19, 20, $22,23,28,30,32,35,36,38$; near stream, on wet soil, on wet rock.

P. seriata Mitt. - Loc.: 9, 22; near stream, on wet soil.

$P$. tomentella Molendo - Loc.: 10, 31; near stream, on wet soil, on wet rock.

Bryaceae Schwagr.

Bryum Hedw.

Bryum argenteum Hedw. - Loc.: 13, 14, 16, 38; on soil.

B. dichotomum Hedw. - Loc.: 11, 31; on soil.

B. gemmiparum De Not. - Loc.: 13; on soil.

B. schleicheri DC. - Loc.: 5, 9, 19, 20, 26, 30, 36; near stream, on wet soil.

Imbribryum N.Pedersen

Imbribryum alpinum (Huds. ex With.) N. Pedersen Loc.: 2, 7, 13, 16, 17, 32; on wet soil.

Ptychostomum Hornsch.

Ptychostomum capillare (Hedw.) Holyoak \& N. Pedersen. - Loc.: 22, 35; on soil.

P. creberrimum (Taylor) J.R. Spence \& H.P. Ramsay (Syn: Bryum creberrimum

Taylor)-Loc.: 2, 9, 11, 12, 17; on soil.
P. donianum (Grev.) Holyoak \& N. Pedersen. - Loc.: 18, 35; on soil.

P. elegans (Nees) D.Bell \& Holyoak - Loc.: 1, 7, 19, 20, 21, 23, 36, 40; on soil.

P. imbricatulum (Müll. Hal.) Holyoak \& N. Pedersen.

- Loc.: 6, 13, 17, 19, 24, 30, 31, 32, 36; on soil.

P. moravicum (Podp.) Ros \& Mazimpaka. - Loc.: 1, 2,

$6,7,8,12,16,19,20,34,38,39$; on soil, on tree brak.

P. pallens (Sw.) J.R. Spence - Loc.: 7, 9; on soil.

\#P. pallescens (Schleich. ex Schwägr.) J.R. Spence Loc.: 29, 38; on soil.

P. pseudotriquetrum var. bimum (Schreb.) Holyoak \& N. Pedersen - Loc.: 11, 19, 25, 30, 38, 39; near stream, on wet soil.

P. pseudotriquetrum (Hedw.) J.R. Spence \& H.P. Ramsay var. pseudotriquetrum - Loc.: 5, 8, 9, 10, 11, $12,18,19,20,22,23,25,28,29,30,31,35,36,38,39$; near stream, on wet soil.

P. torquescens (Bruch \& Schimp.) Ros \& Mazimpaka - Loc.: 7; on soil.

\#P. turbinatum (Hedw.) J.R.Spence.- Loc.: 9, 31; on soil.

Rhodobryum (Schimp.) Limpr.

Rhodobryum ontariense (Kindb.) Kindb. - Loc.: 12; on soil.

Mniaceae Schwagr.

Mnium Hedw.

Mnium hornum Hedw. - Loc.: 8; on wet soil.

M. lycopodioides Schwägr. - Loc.: 38.

M. spinosum (Voit) Schwägr. - Loc.: 4, 5, 6, 7, 8, 12, $13,15,16,18,19,20,21,24,29,32,33,34,35,36,39$, 40; on wet soil, on a dead tree trunk.

M. spinulosum Bruch \& Schimp. - Loc.: 16; on wet soil.

M. stellare Hedw. - Loc.: 14; on wet soil.

M. thomsonii Schimp. - Loc.: 1, 3, 35, 39; on wet soil, Plagiomnium T.J.Kop.

Plagiomnium affine (Blandow ex Funck) T.J.Kop. Loc.: 1, 7, 8, 13, 16, 29, 32; on soil, on wet soil.

P. cuspidatum (Hedw.) T.J.Kop. - Loc.: 1, 2; on soil.

P. elatum (Bruch \& Schimp.) T.J. Kop. - Loc.: 13, 14, 18, 30, 32, 35; on soil.

P. ellipticum (Brid.) T.J.Kop. - Loc.: 10, 14, 16, 17, 31, 38, 39; on wet soil.

P. medium (Bruch \& Schimp.) T.J.Kop. - Loc.: 6, 10, 18, 21, 29, 30, 31, 35, 38, 39, 40; on soil.

P. undulatum (Hedw.) T.J.Kop. - Loc.: 1, 4, 7, 8, 12, 13, 18, 19, 20, 21, 28, 29, 30, 32, 35, 36, 39, 40; on soil, on rock.

Pohlia Hedw.

Pohlia cruda (Hedw.) Lindb. - Loc.: 3, 9, 12, 14, 15, 16, 38, 39; on soil.

\#P. melanodon (Brid.) A.J. Shaw - Loc.: 16, 39; on wet soil.

P. nutans (Hedw.) Lindb. - Loc.: 12, 38; on wet soil.

P. wahlenbergii (F.Weber \& D.Mohr) A.L.Andrews. -

Loc.: 1, 8, 12, 16; on wet soil.

Rhizomnium (Broth.) T.J.Kop.

Rhizomnium magnifolium (Horik.) T.J.Kop. - Loc.: 2, $3,10,22,29,31,35$; on wet soil. 
R. pseudopunctatum (Bruch \& Schimp.) T.J. Kop. Loc.: 9, 26; on wet soil.

R. punctatum (Bruch \& Schimp.) T.J.Kop. - Loc.: 1, 6, 7, 8, 9, 10, 16, 18, 19, 20, 21, 22, 29, 30, 31, 35, 36, 37, 39,40 ; on wet soil, on a dead tree trunk..

Orthotrichaceae Arn.

Lewinskya F.Lara, Garilleti \& Goffinet

Lewinskya affinis (Schrad. ex Brid.) F.Lara, Garilleti \& Goffinet - Loc.: 11, 16, 39; on tree bark.

L. rupestris (Schleich. Ex Schwägr.) F.Lara, Garilleti \& Goffinet. - Loc.: 4, 11, 13,

$15,25,30,32,33$; on rock, on tree bark.

L. speciosa (Nees) F.Lara, Garilleti \& Goffinet. - Loc.:

3, 8, 13, 14, 32, 38, 39; on tree bark.

L. striata (Hedw.) F.Lara, Garilleti \& Goffinet - Loc.: 16, 39; on tree bark.

Nyholmiella Holmen \& E.Warncke

\#Nyholmiella obtusifolia (Brid.) Holmen \&

E.Warncke. - Loc.: 16; on tree bark.

Orthotrichum Hedw.

\#Orthotrichum alpestre Hornsch. Ex Bruch \& Schimp.

- Loc.: 3, 13, 14, 17, 30, 39.

\#O. anomalum Hedw. - Loc.: 38; on rock.

\#O. tenellum Bruch ex Brid - Loc.: 17, 31; on rock.

Pulvigera Plášek, Sawicki \& Ochyra

\#Pulvigera lyellii (Hook. \& Taylor) Plášek, Sawicki \&

Ochyra - Loc.: 7; on tree bark.

Ulota D.Mohr

Ulota crispula Bruch. - Loc.: 1, 2, 3, 4, 8, 12, 16, 39;

on tree bark.

Fontinalaceae Schimp.

Fontinalis Hedw.

Fontinalis antipyretica Hedw. - Loc.: 1, 23; submerged.

Plagiotheciaceae (Broth.) M.Fleisch.

Herzogiella Broth.

\#Herzogiella seligeri (Brid.) Z.Iwats. - Loc.: 1, 4, 5, 6, 8, 18, 21, 34, 35, 40; on a dead tree trunk.

Plagiothecium Schimp.

Plagiothecium cavifolium (Brid.) Z. Iwats. - Loc.: 1, 12; on soil, on a dead tree trunk.

P. denticulatum (Hedw.) Schimp. - Loc.: 18, 35, 39; on soil, on a dead tree trunk.

\#P. latebricola Schimp. - Loc.: 16; on soil.

P. laetum Schimp. - Loc.: 2, 26; on soil.

P. succulentum (Wilson) Lindb. - Loc.: 2, 3, 6, 8, 12,

16, 21, 26, 38, 40; on soil.

Pterigynandraceae Schimp.

Pterigynandrum Hedw.

Pterigynandrum filiforme Hedw. - Loc.: 1, 2, 3, 4, 5, 6, $8,13,15,16,18,19,20,21,29,32,33,34,35,36,38$, 39,40 ; on rock, on tree bark.

Climaciaceae Kindb.

Climacium F. Weber \& D. Mohr.

Climacium dendroides (Hedw.) F. Weber \& D. Mohr. - Loc.: 6, 9, 10, 12, 13, 14, 16, 18, 19, 20, 26, 29, 30,

31, 32, 35, 36, 37, 39; on wet soil.

Amblystegiaceae Kindb.

Campylium (Sull.) Mitt.
Campylium protensum (Brid.) Kindb. - Loc.: 9, 10, 11, 19, 20, 22, 23, 24, 25, 29, 30, 31, 35; on wet soil, on wet rock.

Campylophyllopsis W.R. Buck

\#Campylophyllopsis calcarea (Crundw. Ve Nyholm)

Ochyra (Syn: Campylophyllum alcareum (Mitt.) Hedenäs.) - Loc.: 12, 22, 24; on wet soil.

Cratoneuron (Sull.) Spruce

Cratoneuron filicinum (Hedw.) Spruce. - Loc.: 1, 2, 4, $5,10,11,17,19,20,28,29,30,31,36$; on wet soil, on wet rock.

Drepanium (Schimp.) C.E.O.Jensen

Drepanium fastigiatum (Hampe) C.E.O.Jensen (Syn: Hypnum recurvatum (Lindb. \& Arnell) Kindb. - Loc.: 29; on rock.

Hygroamblystegium Loeske

\#Hygroamblystegium fluviatile (Hedw.) Loeske - Loc.:

19, 20; near stream, on wet soil.

Hygrohypnum Lindb.

Hygrohypnum luridum (Hedw.) Jenn. - Loc.: 2; near stream, on wet soil.

Palustriella Ochryra

Palustriella falcata (Brid.) Hedenäs. - Loc.: 1, 12, 22 , 24, 28, 29, 30; on wet soil.

Platyhypnum Loeske

Platyhypnum duriusculum (De Not.) Ochyra (Syn: Hygrohypnum duriusculum (De Not.) D.W.Jamieson)

- Loc.: 11, 38; near stream, on wet soil.

Pseudoamblystegium Vanderp. \& Hedenäs

Pseudoamblystegium subtile (Hedw.) Vanderp. \& Hedenäs - Loc.: 3; on tree bark.

Scorpidiaceae Ignatov \& Ignatova

Sanionia Loeske

Sanionia uncinata (Hedw.) Loeske. - Loc.: 1, 2, 3, 5, $6,7,8,9,10,12,14,18,19,21,22,24,26,29,31,35$, $36,37,40$; on soil, on a dead tree trunk.

Calliergonaceae Vanderp., Hedenäs, CJCox \& AJShaw

Warnstorfia Loeske

\#Warnstorfia fluitans (Hedw.) Loeske - Loc.: 23; near stream, on wet soil.

Pseudoleskeaceae Schimp.

Lescuraea Schimp.

Lescuraea incurvata (Hedw.) E. Lawton. - Loc.: 15, 19, 20, 22, 24, 30, 33, 38; on rock.

L. mutabilis (Brid.) Lindb. ex I. Hagen. - Loc.: 10, 12, 24, 31; on tree bark.

\#L. plicata (Schleich. ex F. Weber \& D. Mohr) Broth. - Loc.: 15, 33; on soil.

Pseudoleskeellaceae Ignatov \& Ignatova

Pseudoleskeella Kindb.

Pseudoleskeella nervosa (Brid.) Nyholm. - Loc.: 1, 2, $3,4,6,7,10,12,14,15,16,31,33,34,38,39$; on tree bark.

Thuidiaceae Schimp.

Abietinella Müll.Hal.

Abietinella abietina (Hedw.) M.Fleisch. var. abietinaLoc.: 8, 14, 17, 23, 30, 31, 36; on soil, on rock. 
A. abietina (Hedw.) M.Fleisch. var. hystricosa (Mitt.) Sakurai. - Loc.: 15, 16, 19, 20, 22, 26, 33, 38; on soil, on rock.

Thuidium Schimp.

Thuidium assimile (Mitt.) A.Jaeger. - Loc.: 1, 2, 6, 10, $15,16,22,26,31,33,37,39$; on soil, on rock, on a dead tree trunk.

T. delicatulum (Hedw.) Schimp. - Loc.: 9; on soil.

T. recognitum (Hedw.) Lindb. - Loc.: 19, 23; on soil.

T. tamariscinum (Hedw.) Schimp. - Loc.: 1, 2, 7, 12,

$21,28,29,31,35,40$; on soil, on rock, on a dead tree trunk.

Brachytheciaceae Schimp.

Brachytheciastrum Ignatov \& Huttunen

Brachytheciastrum velutinum (Hedw.) Ignatov \&

Huttunen. - Loc.: 1, 2, 3, 5, 8, 16, 19, 20, 21, 40; on soil, on a dead tree trunk.

Brachythecium Schimp.

Brachythecium albicans (Hedw.) Schimp. - Loc.: 5, 6, 12, 13, 14, 19, 20, 32, 34, 36; on soil.

B. campestre (Müll.Hal.) Schimp. - Loc.: 1; on soil. \#B. cirrosum (Schwägr.) Schimp - Loc.: 35; on soil B. glareosum (Bruch ex Spruce) Schimp. - Loc.: 2; on soil.

B. laetum (Brid.) Schimp. - Loc.: 1, 2, 4, 5, 16, 32; on soil

B. mildeanum (Schimp.) Schimp. Ex Milde - Loc.: 2; on soil.

B. rivulare Schimp. - Loc.: 1, 2, 3, 5, 6, 9, 10, 11, 12, $13,14,15,18,19,20,22,24,26,28,29,30,31,32,33$, $35,36,37,38,39$; near stream, on wet soil.

B. rutabulum (Hedw.) Schimp. - Loc.: 1, 9; near stream, on wet soil.

B. salebrosum (Hoffm. ex F. Weber \& D. Mohr) Schimp. - Loc.: 2, 3; on soil.

\section{Cirriphyllum Grout}

Cirriphyllum crassinervium (Taylor) Loeske \& M.Fleisch. - Loc.: 1, 2; on soil, on rock.

C. piliferum (Hedw.) Grout - Loc.: 12; on soil.

Eurhynchiastrum Ignatov \& Huttunen

Eurhynchiastrum pulchellum (Hedw.) Ignatov \& Huttunen. - Loc.: 15, 38; on soil.

Eurhynchium Schimp.

Eurhynchium angustirete (Broth.) T.J.Kop. - Loc.: 1, $2,3,6,7,8,16,18,19,20,21,28,29,35,36,40$; on soil.

E. striatum (Hedw.) Schimp. - Loc.: 6, 39; on soil.

Homalothecium Schimp.

Homalothecium lutescens (Hedw.) H.Rob. - Loc.: 15, 38; on soil, on rock, on a dead tree trunk.

H. philippeanum (Spruce) Schimp. - Loc.: 14, 30, 38; on soil, on rock.

H. sericeum (Hedw.) Schimp. - Loc.: 14, 17; on soil, on rock.

Kindbergia Ochyra

Kindbergia praelonga (Hedw.) Ochyra - Loc.: 2, 39; on soil.

Oxyrrhynchium (Schimp.) Warnst.

Oxyrrhynchium hians (Hedw.) Loeske. - Loc.: 1, 3, 8; on soil, on rock.
O. speciosum (Brid.) Warnst. - Loc.: 12; on soil.

Plasteurhynchium M.Fleisch. ex Broth.

Plasteurhynchium striatulum (Spruce) M. Fleisch. -

Loc.: 10, 29, 31; on soil.

Pseudoscleropodium (Limpr.) M.Fleisch.

Pseudoscleropodium purum (Hedw.) M.Fleisch. Loc.: 11, 12; on soil.

Rhynchostegium Schimp.

Rhynchostegium riparioides (Hedw.) Cardo - Loc.: 2; on wet rock.

R. megapolitanum (Blandow ex F.Weber \& D.Mohr) Schimp. - Loc.: 1; on soil.

Sciuro-hypnum Hampe

Sciuro-hypnum flotowianum (Sendtn.) Ignatov \& Huttunen - Loc.: 2; on soil.

S. populeum (Hedw.) Ignatov \& Huttunen - Loc.: 17; on rock.

Hypnaceae Schimp.

Hypnum Hedw.

Hypnum andoi A.J.E.Sm. - Loc.:1, 2, 16, 17, 28, 34, 39; on rock, on tree bark.

H. bambergeri Schimp. - Loc.: 1, 4, 5, 14, 31, 34; on rock.

H. callichroum Brid. - Loc.: 8; on rock.

H. cupressiforme var. cupressiforme Hedw. - Loc.: 1, $2,4,5,6,7,8,13,15,16,17,18,19,20,23,28,29,32$,

$33,35,36,39$; on soil, on rock, on tree bark, on a dead tree trunk.

H. cupressiforme var. filiforme Brid. - Loc.: 1, 3, 5, 6, 7, 12, 16, 18, 29, 34; on soil, on tree bark.

H. cupressiforme var. lacunosum Brid. - Loc.: 1, 7, 10, 13, 14, 15, 23, 31, 32, 38; on soil, on rock.

\#H. hamulosum Schimp. - Loc.: 5, 11, 14, 16, 29; on soil.

H. jutlandicum Holmen \& E.Warncke. - Loc.: 6, 16; on soil, on a dead tree trunk.

H. resupinatum Taylor (Syn: Hypnum cupressiforme var. resupinatum (Taylor) Schimp. - Loc.: 1, 2, 4, 6, 8, $11,15,16,25,28,29,33,34,39$; on soil, on tree bark. Pylaisiadelphaceae Goffinet \& W.R.Buck

Platygyrium Schimp.

Platygyrium repens (Brid.) Schimp. - Loc.: 19, 20; on rock, on soil.

Pylaisiaceae Schimp.

Buckia D.Rios, M.T.Gallego \& J.Guerra

Buckia vaucheri (Lesq.) D.Rios, M.T.Gallego \& J.Guerra (Syn: Hypnum vaucheri Lesq.) - Loc.: 8; on soil.

Calliergonella Loeske

Calliergonella cuspidata (Hedw.) Loeske. - Loc.: 1, 2 , 9, 10, 12, 13, 16, 19, 20, 22, 23, 29, 30, 31, 32, 36, 39; near stream, on wet soil.

C. lindbergii (Mitt.) Hedenäs. - Loc.: 1, 19, 35; on wet soil.

Homomallium (Schimp.) Loeske

\#Homomallium incurvatum (Schrad . ex Brid.) Loeske - Loc.:10, 12, 19, 20, 31; on rock.

Pylaisia Schimp.

Pylaisia polyantha (Hedw.) Schimp. - Loc.: 1, 19, 20; on tree bark. 
Roaldia P.E.A.S.Câmara \& Carv.-Silva

Roaldia revoluta (Mitt.) P.E.A.S.Câmara \& M.Carvalho-Silva (Syn: Hypnum revolutum (Mitt.) Lindb. - Loc.: 1, 2, 8, 14, 16, 19, 20, 30, 34, 36, 39; on rock

Hylocomiaceae M. Fleisch.

Hylocomiadelphus Ochyra \& Stebel

Hylocomiadelphus triquetrus (Hedw.) Ochyra \& Stebel (Syn: Rhytidiadelphus triquetrus (Hedw.) Warnst.) Loc.: 7, 8, 9, 11, 12, 16, 18, 22, 25, 29, 35, 39; on soil. Hylocomium Schimp.

Hylocomium splendens (Hedw.) Schimp. - Loc.: 4, 7, 8, 11, 12, 15, 17, 18, 19, 20, 21, 25, 29, 33, 35, 36, 39, 40; on soil, on rock.

Pleurozium Mitt.

Pleurozium schreberi (Brid.) Mitt. - Loc.: 4, 5, 8, 11, 18, 19, 20, 21, 22, 25, 34, 35, 36, 39, 40; on soil.

Rhytidiadelphus (Limpr.) Warnst.

Rhytidiadelphus squarrosus (Hedw.) Warnst. - Loc.: 29, 35; on soil.

R. subpinnatus (Lindb.) T.J. Kop. - Loc.: 19, 20, 26; on soil.

Rhytidiaceae Broth.

Rhytidium (Sull.) Kindb.

Rhytidium rugosum (Ehrh. ex Hedw.) Kindb. - Loc.:

$11,13,14,15,17,25,32,33,38$; on soil, on rock.

Entodontaceae Kindb.

Entodon Müll. Hal.

Entodon concinnus (De Not.) Paris. - Loc.: 1, 2, 14, 15, 16, 17, 19, 20, 33, 36, 38; on soil.

Leucodontaceae Schimp.

Leucodon Schwägr.

Leucodon sciuroides (Hedw.) Schwägr. - Loc.: 1, 2, 3, $5,6,12,14,15,16,17,29,33,34,39$; on rock, on tree bark.

Neckeraceae Schimp.

Alleniella S.Olsson, Enroth \& D.Quandt

Alleniella complanata (Hedw.) S.Olsson, Enroth \& D.Quandt. - Loc.: 1, 2, 3, 6, 12, 21, 29, 34, 40; on tree bark.

Neckera Hedw.

+Neckera pumila Hedw. - Loc.: 7; on tree bark.

Thamnobryum Nieuwl.

Thamnobryum alopecurum (Hedw.) Gangulee. - Loc.: 8, 19, 20; on wet soil, on wet rock.

Pseudanomodon (Limpr.) Ignatov \& Fedosov

Pseudanomodon attenuatus (Hedw.) Ignatov \& Fedosov (Syn: Anomodon attenuatus (Hedw.)

Huebener) - Loc.: 2, 28; on soil.

Lembophyllaceae Broth.

Isothecium Brid.

Isothecium alopecuroides (Lam. ex Dubois) Isov. Loc.: 1, 2, 3, 4, 5, 7, 8, 12, 15, 16, 18, 19, 20, 21, 23, 28, 29, 34, 35, 39, 40; on soil, on rock.

Myuriaceae M.Fleisch

Ctenidium (Schimp.) Mitt.

Ctenidium molluscum (Hedw.) Mitt. - Loc.:1, 7, 11, 12, $16,22,25,28,29$; on soil, on rock.

Anomodontaceae Kindb.

Anomodon Hook. \& Taylor
Anomodon rugelii (Müll. Hal.) Keissl. - Loc.: 1; on soil.

A. viticulosus (Hedw.) Hook. \& Taylor - Loc.: 2, 29; on soil, on rock.

\subsection{Discussion}

Families with the highest number of species in the study area are Grimmiaceae (30), Brachytheciaceae (27), Pottiaceae (21), Mniaceae (19), Bryaceae (18), Orthotrichaceae (10), Hypnaceae (9), Amblystegiaceae (15), Polytrichaceae (9), Bartramiaceae (8), Sphagnaceae (7), Scapaniaceae (7), Anastrophyllaceae (6), Thuidiaceae (6), Pylaisiadelphaceae (6), Hylocomiaceae (5), Plagiotheciaceae (5), Dicranaceae (5), Lophocoleaceae (5), Neckeraceae (4), Lophoziaceae (4), Solenostomataceae (3), Plagiochilaceae (3), Encalyptaceae (3), Fissidentaceae (3), Rhabdoweisiaceae (3), Pseudoleskeaceae (3), respectively.

Grimmia (12), Schistidium (11), Brachythecium (9), Hypnum (9), Racomitrium (7), Sphagnum (7), Mnium (6), Plagiomnium (6), Dicranum (5), Syntrichia (5), Philonotis (5) and Plagiothecium (5), Scapania (4), Barbilophozia (4), Solenostoma (3) and Lophocolea (3) are the most common genera in the study area. Moreover the genus of Grimmia has the biggest diversity among others genus (represented by 12 species, growing on rocks mostly in study area), second common genus is Schistidium (represented by 11 species, mostly on rocks). The other common genera are Brachythecium (9 members, mostly on rocks, soil, bark of coniferous and deciduous trees), Hypnum (9 members, mostly on rocks, soil, bark of coniferous and deciduous trees), Racomitrium (7 members, mostly on rocks), Sphagnum (7 members, mostly submerged), Mnium (6 members, mostly on wet soil), Plagiomnium (6 members, mostly on wet soil), Dicranum (5 members, mostly on soil, rock, dead tree trunk), Syntrichia (5 members, mostly on soil), Philonotis (5 members, mostly submerged ) and Plagiothecium (5 members, mostly on rocks, soil, bark of trees), Scapania (4 members, mostly on rocks and soil), Barbilophozia (4 members, mostly on rocks and soil), Solenostoma and Lophocolea consisting of 3 members.

Rhynchostegium riparioides, Hypnum callichroum, Sciuro-hypnum flotowianum, Rhynchostegium riparioides, Oxyrrhynchium speciosum, Cirriphyllum piliferum, Thuidium delicatulum, Warnstorfia fluitans, Hygrohypnum luridum, Plagiothecium latebricola, Nyholmiella obtusifolia, Bryum gemmiparum, Bartramia pomiformis, Schistidium helveticum, Grimmia muehlenbeckii, Pseudephemerum nitidum, Sphagnum inundatum, Calypogeia fissa, Diplophyllum albicans, Cephaloziella divaricata, Gymnocolea inflata, Barbilophozia hatcheri are very rare species in the study area. 
Neckera pumila, Pseudephemerum nitidum new record for A4 square, 52 taxa new for Giresun Province and all taxa new for Kümbet high plateau (Dereli district) (Özdemir and Batan, 2017b; Erata et al., 2018).

Barbilophozia lycopodioides, B. sudetica, Lophozia ventricosa, Tritomaria exsecta, Trilophozia quinquedentata, Scapania aequiloba, S. subalpina, Solenostoma hyalinum Blepharostoma trichophyllum, Lepidozia reptans, Riccardia multifida, R. palmata, Sphagnum fallax, S. platyphyllum, S. squarrosum, $S$. teres, Atrichum tenellum, Polytrichastrum alpinum, Encalypta ciliata, Flexitrichum flexicaule, F. gracile, Dicranella rufescens, Fissidens taxifolius, Dicranum spadiceum, Tortella humilis T. nitida, Tortula mucronifolia, Weissia brachycarpa, Saelania glaucescens, Grimmia anomala, G. elatior, G. funalis, Schistidium dupretii, S. helveticum, Bartramia ithyphylla, Ptychostomum pallescens, P. turbinatum, Pohlia melanodon, Nyholmiella obtusifolia, Orthotrichum alpestre, O. anomalum, O. tenellum, Pulvigera lyellii, Herzogiella seligeri, Plagiothecium latebricola, Campylophyllopsis calcarea, Hygroamblystegium fluviatile, Warnstorfia fluitans, Lescuraea plicata, Brachythecium cirrosum, Hypnum hamulosum and Homomallium incurvatum are new to Giresun Province (Özdemir and Batan, 2017b; Erata et al., 2018).

These are the some studies carried out in Giresun province (Handel-Mazzetti, 1909; Ünal, 1973; Özdemir and Baydar, 1997; Özdemir, 1999; Özdemir, 2001a, b; Özdemir and Koz, 2005, 2006, 2007; Özdemir and Batan, 2008; Özdemir, 2008; Kırmacı and Kürschner, 2013; Erata et al., 2018). As a results of these studies, in a total 367 bryophyte taxa have been reported in Giresun province. The first bryofloristic studies in the Dereli district of Giresun province was carried out by Özdemir and Koz (2007). In this study, mosses of the area were investigated and 91 taxa were determined in this study.

The present study 52 new bryophyte (40 mosses and 12 liverworts) taxa adds to the bryophyte flora of Giresun province. This study has the highest number of species that has been done in Giresun province so far. Finally, the number of bryophyte taxa of Giresun province reached 419 with the present paper.

\section{Acknowledgements}

The authors are grateful to the Çanakkale Onsekiz Mart University for financial support (FBA-2019-2950 coded Project). We also thank Öznur ÖZEN for helping our determination of Sphagnum species.

\section{Reference}

Anşin R. 1981. Main vegetation types inner and coastal part of east black sea region. Journal of the Foresty Facultry of the Karadeniz Technical University. 4:1, 14-25.
Blom H.H. 1996. A Revision of the Schistidium apocarpum Complex in Norway and Sweden, ISBN: 3-443-62021-3. Bryophytorum Bibliotheca. Band.

Brugués M. Cros R.M. Guerra J. 2007. Flora Briofitica Ǵbérica Volume I, Uniersidad de Murcia. Sociedad Espanola de Briyologia Murcia. Murcia.

Crum A.H. Anderson E.L. 1981. Mosses of Eastern North America. Columbia University Press. Newyork.

Erata H. Batan N. Özdemir T. 2018. The Bryophyte Flora of Sis Mountain (Giresun-Trabzon, Turkey). Anatolian Bryology. 4:1, 46-64.

Erata H. Özen Ö. Batan N. Alataş M. 2021. Pohlia Hedw. and Oleolophozia L.Söderstr., De Roo \& Hedd. species new to Turkey and South-West Asia. Cryptogamie Bryologie. 42:1, 1-10.

Frey W. Frahm J.P. Fischer E. Lobin W. 2006. The liverworts, mosses and ferns of Europe. Essex. Harley Books.

Greven H.C. 2003. Grimmias of The World. Backhuys Publishers. Leiden.

Guerra J. Cano M.J. Cros R.M. 2006. Flora Briofitica Ibérica VoIume 3. Uniersidad de Murcia, Sociedad Espanola de Briyologia Murcia. Murcia.

Guerra J. Brugués M. Cano M.J. Cros R.M. 2010. Flora Briofitica Ibérica VoIume 4. Uniersidad de Murcia, Sociedad Espanola de Briyologia Murcia. Murcia.

Guerra J. Cano M.J. Brugués M. 2014. Flora Briofitica Ibérica VoIume 5, Uniersidad de Murcia, Sociedad Espanola de Briyologia Murcia, Murcia.

Guerra J. Cano M.J. Brugués M. 2018. Flora Briofitica Ibérica VoIume 6, Uniersidad de Murcia, Sociedad Espanola de Briyologia Murcia, Murcia.

Handel-Mazzetti H.M. 1909. Ergebnisse einer botanische Reise in des Pontische Randgebirge in Sandchak Trapezunt. Ann. Nathist, Hofmus. 23: $124-212$.

Heyn C.C. Herrnstadt I. 2004. The Bryophyte Flora of Israel and Adjacent Regions. The Israel Academy of Sciences and Humanities. Oron.

Hodgetts N.G. Söderström L. Blockeel T.L. Caspari S. Ignatov M.S. Konstantinova N.A. Lockhart N. Papp B. Schröck C. SimSim M. ve ark. 2020. An annotated checklist of bryophytes of Europe, Macaronesia and Cyprus. Journal of Bryology. 42:1, 1-116.

Kırmacı M. Kürschner H. 2013. The genus Sphagnum L. in Turkey with $S$. contortum, S. fallax, $S$. magellanicum and $S$. rubellum new to Turkey and Southwest Asia. Nova Hedwigia. 96: 383397.

Kürschner H. Frey W. 2011. Liverworts, mosses and hornworts of Southwest Asia (Marchantiophyta, 
Bryophyta, Anthocerotophyta). Nova Hedwigia. 139: 1-240.

Lewinsky J. 1993. A synopsis of the genus Orthotrichum Hedw. (Musci, Orthotrichaceae). Bryobrothera. 2: 1-59.

Paton J. 1999. The Liverworts Flora of the British Isles, ISBN: 0-946589-60-7, 626 pp, Harley Books. England.

Pedrotti C.C. 2001. Flora dei muschi d'Italia (Sphagnopsida, Andreaeopsida, Bryopsida, I parte). Antonio delfino Editore medicinascienze. Roma.

Pedrotti C.C. 2006. Flora dei muschi d'Italia. Bryopsida (II parte). Antonia Delfi no Editore medicina-scienze, Roma.

Nyholm E. 1986. Illustrated Flora of Nordic Mosses, Fasc. 1. Fissidentaceae - Seligeriaceae, 1-72, The Nordic Bryological Society, Lund.

Nyholm E. 1989. Illustrated Flora of Nordic Mosses, Fasc. 2. Pottiaceae - SplachnaceaeSchistostegaceae, 75-141, The Nordic Bryological Society, Lund.

Nyholm E. 1993. Illustrated Flora of Nordic Mosses, Fasc. 3. Bryaceae-Rhodobryaceae Mniaceae Cinclidiaceae-Plagiomniaceae. 145-244, The Nordic Bryological Society, Lund.

Nyholm E. 1998. Illustrated Flora of Nordic Mosses, Fasc. 4. Aulacomniaceae- MeesiaceaeCatocopiaceae-Bartramiaceae-TimmiaceaeEncalyptaceae-Grimmiaceae PtychomitriaceaeHedwigiaceae-Orthotrichaceae. 145-244, The Nordic Bryological Society, Lund.

Özdemir T. 1999. Some taxa of Bryophyta spreaded In Eynesil district (Giresun-Turkey). Energy, Education, Science and Technology. 4: 30-41.

Özdemir T. 2001a. A new record for the moss flora of Turkey, Tortella inflexa (Bruch) Brot. Turkish Journal of Botany. 25: 365-366.
Özdemir T. 2001b. The bryophyta flora of Giresun province centre and near vicinity. Turkish Journal of Botany. 25: 275-283.

Özdemir T. 2008. Rhytidiadelphus loreus (Hedw.) Warnst. (Hylocomiaceae, Bryopsida), new to the moss flora of Turkey and south-west Asia. Cryptogamie Bryologie. 29: 207-208.

Özdemir T. Baydar S. 1997. Some taxa of Bryophyta in the Tirebolu District (Giresun). Turkish Journal of Botany. 21: 335-339.

Özdemir T. Koz B. 2005. The moss flora of Bulancak (Giresun) District. Ot Sistematik Botanik Dergisi. 12: 107-116.

Özdemir T. Koz B. 2006. The moss flora of Keşap (Giresun) District. Ot Sistematik Botanik Dergisi. 13: 175-182.

Özdemir T. Koz B. 2007. Contribution to the moss flora of Dereli, Giresun District (Turkey). Acta Botanica Hungarica. 50: 171-180.

Özdemir T. Batan N. 2008. Contributions to the moss flora of Giresun region (Şebinkarahisar And Alucra district). Pakistan Journal of Biological Sciences. 11: 1987-1993.

Özdemir T. Batan N. 2017a. The bryophyte checklist of Trabzon Province of Turkey. Arctoa. 26: 58-67.

Özdemir T. Batan N. 2017b. Bryophyte Checklist of Giresun, North East Turkey. Anatolian Bryology. 3:1, 1-8.

Sesli E. Vizzini A. Contu M. 2015. Lyophyllum turcicum (Agaricomycetes: Lyophyllaceae), a new species from Turkey. Turkish Journal of Botany. 39: 512-519.

Smith A.J.E. 2004. The Moss Flora of Britain and Ireland. Cambridge University Press. Cambridge.

Ünal A. 1973. Türkiye yosunları üzerinde taksonomik bir araştırma. Atatürk Üniversitesi yayınları. No: 116. Sevinç Matbaası, Ankara. 